\title{
Spin waves in magnetic double layers with strong antiferromagnetic interlayer exchange coupling: Theory and experiment
}

\author{
M. Buchmeier,* B. K. Kuanr, R. R. Gareev, D. E. Bürgler, and P. Grünberg \\ Institut für Festkörperforschung, Forschungszentrum Jülich GmbH, D-52425 Jülich, Germany \\ (Received 4 July 2002; revised manuscript received 17 December 2002; published 5 May 2003)
}

\begin{abstract}
We present a simple approach based on continuum theory to calculate spin-wave frequencies in thin magnetic multilayers taking into account both the nonuniform static and dynamic magnetizations, which are present in systems with strong interlayer exchange coupling. The calculation includes in-plane static magnetization, the canted and twisted state, bilinear and biquadratic interlayer exchange coupling, and the dynamic dipolar coupling. Therefore, we are able to compute accurate spin-wave frequencies in strongly antiferromagnetic coupled trilayers over a full hysteresis loop. We consider the field dependence of the spin-wave frequencies of an epitaxial $\mathrm{Fe}(001) / \mathrm{Si}$-wedge/Fe sample with strong antiferromagnetic coupling measured by Brillouin light scattering and find excellent agreement with the model calculation. The fits of the experimental curves verify the existence of the twisted state and allow determining the coupling constants with high precision.
\end{abstract}

DOI: 10.1103/PhysRevB.67.184404

PACS number(s): 75.30.Ds, 75.30.Et, 75.70.-i

\section{INTRODUCTION}

After the discovery of magnetic interlayer exchange coupling in 1986 in Ref. 1, the phenomenon has been explored in much detail, and its origin is now believed to be basically understood. Still some discrepancies remain, for example, the large differences between the theoretically predicted and experimentally observed coupling strengths. ${ }^{2}$ Also, the origin of the biquadratic coupling contribution encountered in most systems remains in discussion. On the other hand, there is an increasing interest in interlayer coupling, not only because it is a new quantum phenomenon, but also due to its applications as artificial antiferromagnets or ferrimagnets in magnetic sensors $^{3}$ or more recently in antiferromagnetically (AFM) coupled storage media for hard disk drives. ${ }^{4}$

Brillouin light scattering (BLS) is a powerful tool to analyze the magnetic properties of thin-film samples, in particular, coupling phenomena. In a BLS experiment the frequencies $\nu_{m}$ of spin waves are measured via inelastic scattering of monochromatic light. The frequency of the photons can be shifted either down or up by $\nu_{m}$ corresponding to the creation (Stokes condition) or annihilation (anti-Stokes condition) of a magnon, respectively. BLS has been extensively used to determine the interlayer exchange coupling in magnetic multilayers. In contrast to static magnetometric methods, BLS also provides the possibility to derive the ferromagnetic (FM) coupling strength without the necessity of spin engineering, ${ }^{5}$ and it is not necessary to analyze the whole remagnetization loop in order to derive the coupling strength. However, to be able to separate bilinear contribution from biquadratic and other nonbilinear contributions to the coupling, the field dependence of the spin-wave frequencies needs to be fitted. On the other hand, the spin-wave frequencies measured in a BLS experiment until now only have been computed for the restricted cases of parallel $(P)$ and antiparallel (AP) alignment ${ }^{6}$ or limited to ultrathin layers with not too strong coupling in the so-called "ultrathin film" approximation (UTFA). ${ }^{7}$ A full treatment in the case of strong coupling, but excluding the biquadratic coupling, has only been done based on quantum mechanic spin-lattice theory, ${ }^{8}$ which is numerically rather elaborate.

Recently, we reported on very strong AF interlayer exchange coupling in excess of $6 \mathrm{~mJ} / \mathrm{m}^{2}$ in epitaxial $\mathrm{Fe} / \mathrm{Si} / \mathrm{Fe}$ trilayer systems. ${ }^{9}$ We will use these systems to compare different modeling schemes. In Sec. I we will discuss the results obtained by the conventional "full" and UTFA approaches for a Fe $(80 \AA)$ /spacer/Fe (100 $\AA$ ) system and demonstrate that these approaches have significant shortcomings when dealing with strong coupling. Moreover, as we show in Sec. II for strongly coupled systems, the magnetization will, in general, not remain uniform, but instead it will twist, forming a partial domain wall parallel to the interface and hence complicate the analysis even more. In Sec. III we introduce a method based on the UTFA, which enables the calculation of frequencies with high precision in strongly coupled multilayers including the twisted state. We call it the "extended ultrathin film approximation" (EUTFA). It can also be used for the treatment of the standing modes and thicker layers, where the UTFA fails. In Sec. IV we describe sample preparation and measurement setup. Finally, in Sec. V we discuss the measured field variation curves for different spacer thicknesses, which we fit with excellent agreement using our EUTFA approach to extract the bilinear and biquadratic coupling strengths versus spacer thickness. Ready-to-use formulas for the conventional multilayer and EUTFA will be provided in the Appendix.

\section{CONVENTIONAL CALCULATION OF THE SPIN-WAVE FREQUENCIES}

Magnon frequencies with finite in-plane wave vector have been calculated for coupled magnetic multilayers with inplane magnetization in the late 1980's by Barnas and Grunberg $^{6}$ and Hillebrands. ${ }^{10}$ We call their method of computing the spin-wave frequencies the "full" approach. They solve the linearized Bloch equation together with the Maxwell equations in the magnetostatic limit $(\operatorname{curl} \vec{H}=0)$ for each individual layer. The solutions are then matched at the interfaces, where, in addition to the magnetostatic boundary 
conditions, (continuity of $H_{\|}$and $B_{\perp}$ ) the Rado-Weertman conditions requiring continuity of the torque density have to be fulfilled. Interface anisotropies and the interlayer coupling enter via boundary conditions. We limit ourselves in the following discussion to the surface-type Damon-Eshbach (DE) modes, which are dominated by the dipolar interaction, lowest in frequency, and approximately uniform in ultrathin films. The so-called standing modes with one or more nodes in the mode profile are not considered in the following, although we will show in Sec. III that their frequency can also be calculated using our EUTFA method.

In analogy with coupled harmonic oscillators (e.g., phonons), the spin-wave modes in coupled double layers can be classified into optic $(O)$ and acoustic $(A)$, depending on whether their frequency depends strongly on the coupling strength or not. ${ }^{11,12}$ We phenomenologically describe the coupling by the corresponding energy density $E_{C}=$ $-J_{1} \cos (\Delta \theta)-J_{2} \cos ^{2}(\Delta \theta)$. In most real samples with AFM coupling corresponding to $J_{1}<0$, there is also a biquadratic contribution favoring $90^{\circ}$ alignment and described by $J_{2}$ $<0$. The optic-mode frequency will then depend on an effective coupling $J_{\text {eff }}=J_{1}+2 J_{2}$ or $J_{\text {eff }}=J_{1}-2 J_{2}$ in the $P$ or AP state, respectively. Therefore, $J_{1}$ cannot be separated from $J_{2}$ by analyzing the $P$ or AP state alone. Moreover, the canted state has to be taken into account if the sample cannot be saturated by the available external field. There are no reports of successfully calculating the spin-wave frequencies in the canted state using the full approach. The dependence of the BLS frequencies on the external field during a remagnetization process is instead usually computed using the UTFA, $, 13,14$ which assumes approximately uniform amplitudes of the spin-wave modes simplifying the calculation a lot as no boundary conditions need to be evaluated. Instead all interactions including the interlayer exchange coupling, surface anisotropy, and the dynamic dipolar coupling are treated as effective volume torques.

In Fig. 1 we compare the frequencies obtained by the full (solid lines) and UTFA approaches (dotted lines) using physical parameters corresponding to our samples with saturation magnetization $M_{s}=1.65 \times 10^{6} \mathrm{~A} / \mathrm{m}$, fourfold anisotropy $K_{1}=45000 \mathrm{~J} / \mathrm{m}^{3}$, gyromagnetic ratio $\gamma / 2 \pi$ $=29.4 \mathrm{GHz} / \mathrm{T}$, (corresponding to a $g$ factor of 2.1), intralayer exchange $A=2 \times 10^{-11} \mathrm{~J} / \mathrm{m}$, and an in-plane wave vector $q=1.67 \times 10^{7} \mathrm{~m}^{-1}$. In Fig. 1(a) the frequencies are plotted as a function of the effective coupling strength $J_{\text {eff }}$ for the AP state with AFM coupling $\left(J_{\text {eff }}<0\right)$ and the $P$ state with FM coupling $\left(J_{\text {eff }}>0\right)$ as defined above. The calculation assumes zero external field and magnetizations aligned along an easy axis of the cubic anisotropy and perpendicular to the in-plane magnon wave vector $\vec{q}$. This situation will be found in experiments when the FM layers have different thickness, the bilinear coupling is dominant, i.e., $\left|J_{1}\right|$ $>2\left|J_{2}\right|$, and a small external field is applied along an easy axis and perpendicular to $\vec{q}$. As can be seen the optic-mode frequency increases with FM and AFM coupling strength in $P$ and AP configurations. While the UTFA predicts the right frequency when the coupling is weak enough, it will overestimate the optic-mode frequency in the case of strong cou-

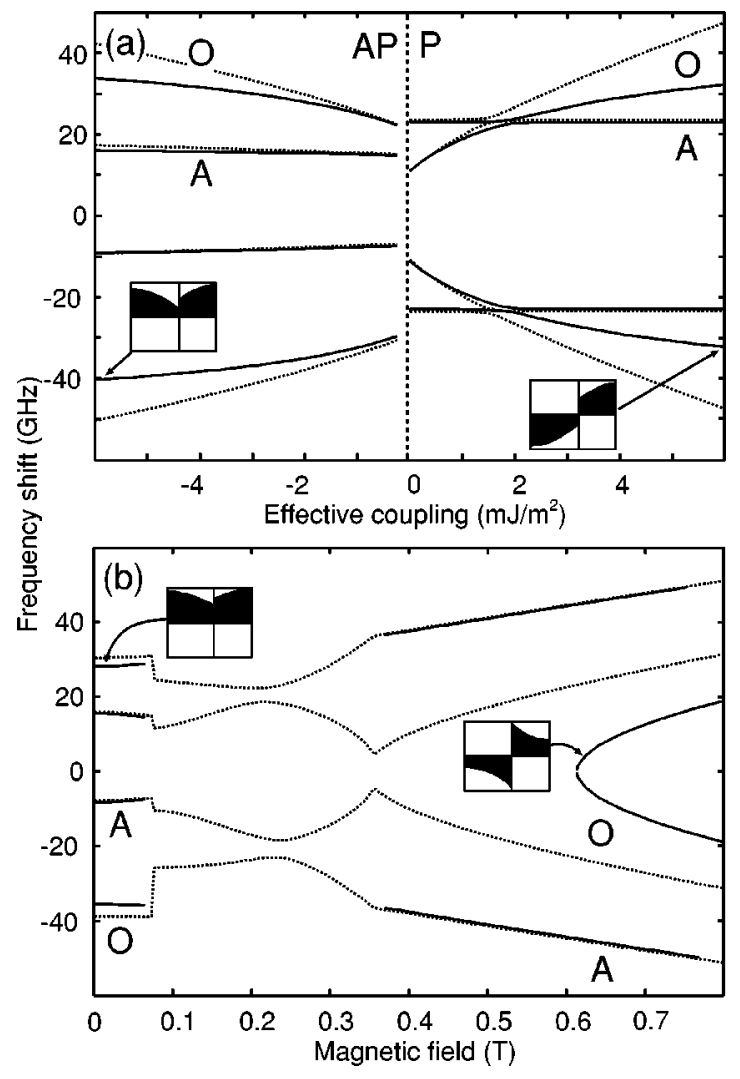

FIG. 1. Calculated acoustic $(A)$ and optic $(O)$ spin-wave frequencies of a $\mathrm{Fe}(001)(80 \AA) /$ spacer/Fe $(100 \AA)$ system. Dotted lines, UTFA; solid lines, full calculation. The insets show the inplane component of the dynamic magnetization as a function of the position. The parameters are given in the text. (a): BLS frequencies in zero field with $\vec{q} \perp \vec{M}$ as a function of effective coupling $J_{\text {eff }}$ defined in the text. (b): BLS frequencies as a function of the external field $B$ applied along the easy axis and with $\vec{q} \perp \vec{B}$.

pling, which, to our knowledge, has not been pointed out in previous publications. The reason for this shortcoming is that, depending on the alignment and sign of the coupling, the optic mode will form a partial node or peak at the interface if the coupling is strong ${ }^{6}$ (see insets in Fig. 1).

Figure 1(b) shows a typical field dependence curve. The angles of the static magnetization, which is assumed to be rigid, correspond to the total minima of the free energy composed of Zeeman energy $E_{Z}$, magnetocrystalline anisotropy energy $E_{a n}$, and interlayer coupling energy $E_{C}$ with coupling constants $J_{1}=-2.6 \mathrm{~mJ} / \mathrm{m}^{2}$ and $J_{2}=-0.2 \mathrm{~mJ} / \mathrm{m}^{2}$ typical for Si thicknesses of $10 \AA$. The external field is applied along the easy axis and perpendicular to $\vec{q}$. At low external field, the magnetizations align AP with the larger magnetic moment in the field direction. When the external field reaches the spin-flop field of about $0.07 \mathrm{~T}$, the magnetizations switch into the canted configuration, and the full calculation cannot be applied any more. The spin flop can be recognized by an abrupt change in the optic and acoustic frequencies. In the rigid magnetization approximation, the sample saturates at a field of about $0.36 \mathrm{~T}$, which shows up as a kink in the acoustic and as a dip in the optic frequency. Interestingly, using the full approach, the optic-mode is not 


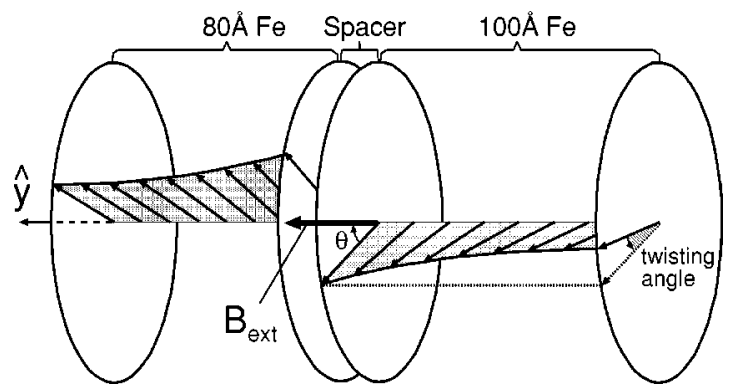

FIG. 2. Schematic view of the twisted magnetization state in a sample with strong AFM coupling. The circles lie in the plane of the interfaces. The in-plane magnetization angle $\theta$ varies in the direction of sample normal $\hat{y}$. The picture corresponds to Fe $(80 \AA) /$ spacer/Fe $(100 \AA)$ with applied field $B_{\text {ext }}=0.5 \mathrm{~T}$ and $J_{1}=-2.6 \mathrm{~mJ} / \mathrm{m}^{2}, J_{2}=-0.2 \mathrm{~mJ} / \mathrm{m}^{2}$ typical for an $\mathrm{Si}$ interlayer thickness of $10 \AA$.

present over a wide field range between about 0.36 and $0.6 \mathrm{~T}$. The reason for the vanishing optic-mode is that the $P$ state with homogeneous magnetization is unstable in this region. Therefore, the assumption of a rigid magnetization is not proper in the case of strong coupling (see Sec. II). The real saturation field instead corresponds approximately to $0.6 \mathrm{~T}$, where the optic-mode appears [compare with Fig. 3(a)].

\section{TWISTED GROUND STATE}

If AFM interlayer coupling and external field are strong enough compared to the intralayer exchange, magnetization will form a partial Bloch-type domain wall parallel to the interface, as sketched in Fig. 2. This is a result of the competing torques exerted at the interface by the coupling and in the bulk by the external field. The twisted magnetization state has been extensively investigated, including BLS, ${ }^{15}$ for so-called exchange springs ${ }^{16}$ and the surface spin-flop phase in antiferromagnets. ${ }^{17,18}$ However, there are only few publications ${ }^{19-21}$ treating the twisted ground state in the case of AFM coupling.

The starting point for calculating the twisted magnetization state is the free energy per unit area:

$$
F=\int\left(E_{e x}+E_{a n}+E_{Z}\right) d y+E_{C}
$$

which is a functional of the in-plane magnetizations angle $\theta(y)$ variable in the direction of the film normal $\hat{y}$. The intralayer exchange term has the form ${ }^{22} E_{e x}=A(\partial \theta / \partial y)^{2}$, and the interlayer coupling depends on the relative magnetization angle at the interfaces $\theta_{i f}: E_{C}=-J_{1} \cos \left(\Delta \theta_{i f}\right)$ $-J_{2} \cos ^{2}\left(\Delta \theta_{i f}\right)$. Finding solution $\theta(y)$, which minimizes the free energy functional, can be done by using calculus of variation as in Ref. 20. However, when treating thin films, it is straight forward to approximate the integral in Eq. (1) by a sum. $\theta(y)$ is defined at $N$ points including interfaces $\theta_{i}$ and assuming linear behavior in between. The volume Zeeman $E_{Z}$ and anisotropy $E_{a n}$ energies can then be approximated by their values using the average angles $\overline{\theta_{i}}=\left(\theta_{i}+\theta_{i+1}\right) / 2$, and the intralayer exchange depends on the relative angle $\Delta \theta_{i}$ $=\theta_{i}-\theta_{i+1}$ between neighboring points:

$$
F \approx \sum_{i \neq i f}\left[\left\{E_{Z}\left(\bar{\theta}_{i}\right)+E_{a n}\left(\bar{\theta}_{i}\right)+A\left(\frac{\Delta \theta_{i}}{\Delta y}\right)^{2}\right\} \times \Delta y\right]+E_{C} .
$$

The term corresponding to the points at the interface ( $i$ $=i f$ ) has to be excluded from the sum. Equation (2) can be easily solved using standard multidimensional minimization algorithms, ${ }^{23}$ at least up to $N \approx 100$.

The possible lower energy in the twisted configuration compared to the uniform magnetization is due to a reduction in the coupling energy at the cost of exchange energy. Taking into account only interfacial points, a critical AFM coupling strength $J_{\text {crit }}$, at which the intralayer exchange and coupling energy will cancel out, can be estimated to be $J_{\text {crit }} \approx$ $-A / d$, where $d$ is the FM layer thickness. For $\left|J_{1}\right| \ll\left|J_{\text {crit }}\right|$, the twisting effects in a thin-film sample can be neglected as the corresponding intralayer exchange will be bigger than the coupling energy. On the other hand, the twist becomes dominant and leads to an disproportionate increase in the saturation field with the coupling strength for $\left|J_{1}\right|>\left|J_{\text {crit }}\right|$ when the anisotropy is negligible. However, if the FM layer thickness $d$ is of the order of the exchange length $\sqrt{A / K_{1}}$, the anisotropy energy $K_{1} d$ becomes important, and the twisted ground state will be suppressed when $J_{1}$ becomes much smaller than $K_{1} d$.

Using $d=100 \AA$ and the literature exchange value for iron of $A=2 \times 10^{-11} \mathrm{~J} / \mathrm{m}$ yields $J_{\text {crit }}=-2 \mathrm{~mJ} / \mathrm{m}^{2}$. The calculated field dependence in Fig. 3 for coupling values of $J_{1}$ $=-2.6 \mathrm{~mJ} / \mathrm{m}^{2}, J_{2}=-0.2 \mathrm{~mJ} / \mathrm{m}^{2}$, and other parameters as in Fig. 1(b) indeed shows pronounced deviations from the uniform state. Although the averaged magnetization angles of the twisted state [solid line in Fig. 3(a)], which approximately determine the frequency of the acoustic mode, are close to the result obtained assuming a rigid static magnetization (dotted line) over a wide field range, they clearly deviate for $B_{\text {ext }}>0.3 \mathrm{~T}$, and the saturation field is about a factor of 2 larger. Another more subtle difference to the rigid approximation is a lowering of the spinflop field. Thus, deriving the coupling strengths from the saturation and spinflop fields, using the rigid approximation, will lead to a systematic overestimation of $J_{1}$ and $J_{2}$.

In Fig. 3(b) we plot the twisting angle defined in Fig. 2 as the relative angle between the magnetizations at the two interfaces of each FM layer. The twisting angle is not negligible apart from the AP state at low fields. It reaches a maximum value of about $20^{\circ}$ where the sample would saturate in the case of a rigid magnetization. Therefore, the optic-mode frequency, which strongly depends on the relative angle at the interlayer, cannot be predicted accurately without including the twist into the calculation.

\section{EXTENDED ULTRATHIN-FILM APPROXIMATION}

The method we use here to calculate the spin-wave frequencies is simple, but predicts right frequencies in the case of strong coupling and even for standing modes. Moreover, it 

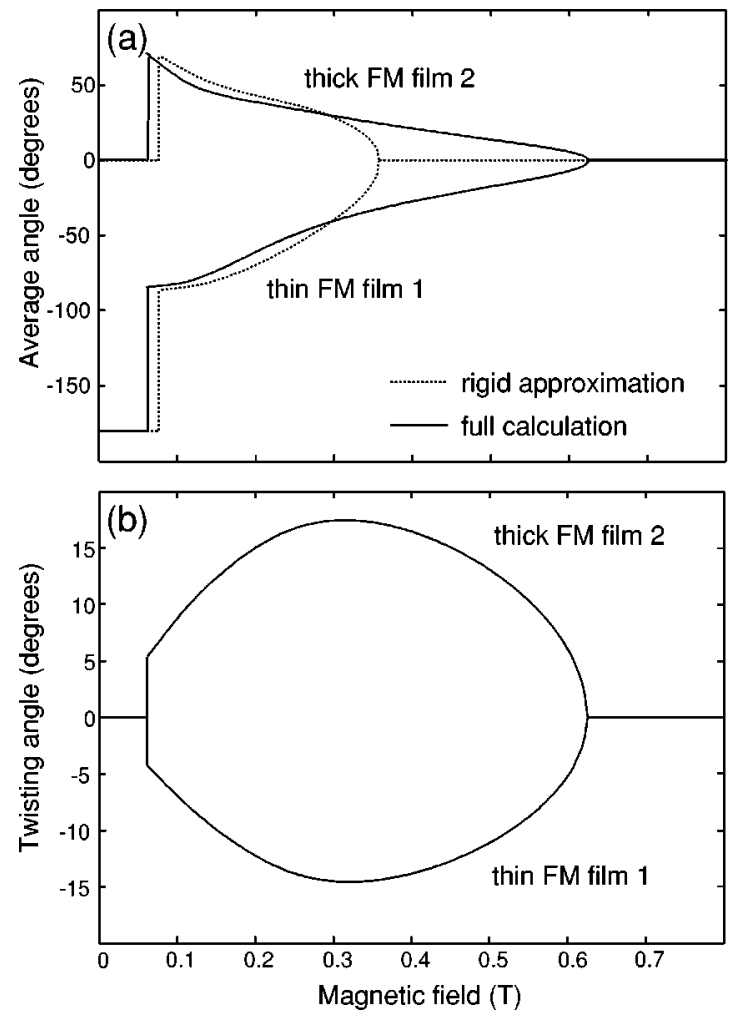

FIG. 3. Calculated field dependence of the twisted state using parameters of Fig. 1. (a) compares the averaged magnetization angles with the rigid magnetization approximation (dotted line). In (b) the twisting angle as defined in Fig. 2 is plotted.

is able to take into account the magnetization twist discussed in the preceding section in a natural way. Having in mind that the UTFA works if only the magnetic layers are thin enough, we virtually subdivide the FM layers into thin sublayers of thickness $\delta$ parallel to the film plane, for which we assume uniform magnetization similar to Grimsditch et al. ${ }^{15}$ If the twisted state is to be taken into account, then the magnetization angle of each sublayer is set to the value obtained from the calculation described in Sec. II. The interlayer exchange coupling and a possible interface anisotropy is taken into account by introducing them in the corresponding interfacial sublayers, but not as a volume contribution of the whole FM layer as in the case of the conventional UTFA.

We treat the intralayer exchange as an effective interlayer coupling between sublayers with a corresponding energy

$$
E_{e x}^{e f f}=\frac{A}{\delta}(\Delta \theta)^{2} \approx-J_{1}^{e f f} \cos \Delta \theta+\mathrm{const} ; \quad J_{1}^{e f f}=\frac{2 A}{\delta},
$$

where the approximation is valid because the relative angle between the magnetizations of two neighboring sublayers $\Delta \theta$ is supposed to be small. The exchange energy has the same form as the bilinear interlayer exchange in the UTFA for multilayers ${ }^{24}$ (full formulas are given in the Appendix), which can readily be used to compute the frequencies of the virtual multilayer stack.

In Fig. 4 we compare the spin-wave frequencies obtained using this method with the full and UTFA approaches as a

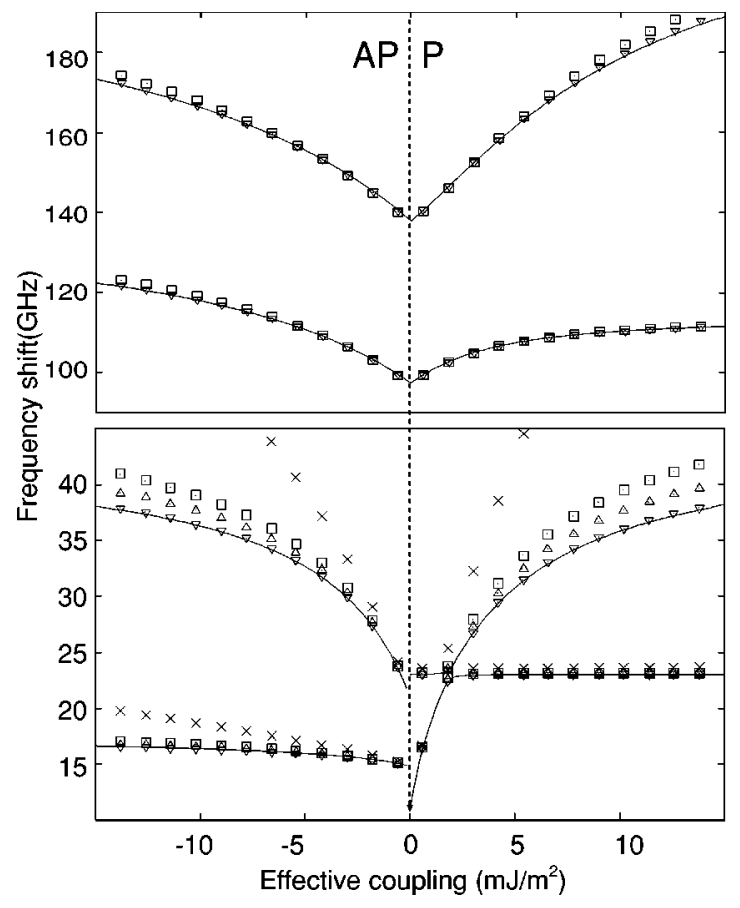

FIG. 4. Convergence of the EUTFA as a function of the coupling strength and the sublayer thickness $\delta$. The solid lines and crosses $(X)$ are the result of the full and conventional UTFA approaches, respectively. Open squares $(\square)$, uptriangles $(\Delta)$, and downtriangles $(\nabla)$ are obtained with EUTFA and correspond to $\delta$ $=20,10$, and $1 \AA$, respectively. All parameters are the same as in Fig. 1(a).

function of the coupling strength without external field similar to Fig. 1(a). The bottom part corresponds to the antiStokes side of Fig. 1(a), however, over a bigger coupling range. The top part shows the lowest standing modes (one node in the mode profile of each magnetic layer), for which the UTFA, of course, cannot be used. Note that the scale of the frequency axis is different in the top and bottom parts of Fig. 4. The frequencies obtained by our EUTFA method converge quickly towards the result of the full approach (solid line) with decreasing $\delta$. While the value of $\delta=20 \AA(\square)$ is already much closer to the full approach than the conventional UTFA $(X)$, a precision better than $1 \mathrm{GHz}$ can be achieved even for the first standing modes using a sublayer thickness of the order of $1 \mathrm{ML}, \delta=1 \AA(\nabla)$.

Apart from the case of strong coupling and the twisted ground state, the method we propose can also be used if the layers are too thick for the conventional UTFA, i.e., when $q d \ll 1$ is not fulfilled. The limiting factor for the validity of the approximation made in the calculation of the dipolar interactions is the thickness $\delta$ of the sublayers and not the thickness $d$ of the FM layers. In Fig. 5 we plot the calculated mode frequencies of a double-layer system as a function of the in-plane wave vector $q$. The 200 - $\AA$-thick Fe layers are FM coupled with $J_{1}=1 \mathrm{~mJ} / \mathrm{m}^{2}$ and a field of $0.1 \mathrm{~T}$ is applied along the easy axis. The conventional UTFA $(X)$ deviates significantly from the full calculation (solid line) for $q d>0.3$, whereas our method is accurate at least up to $q d=4$. 


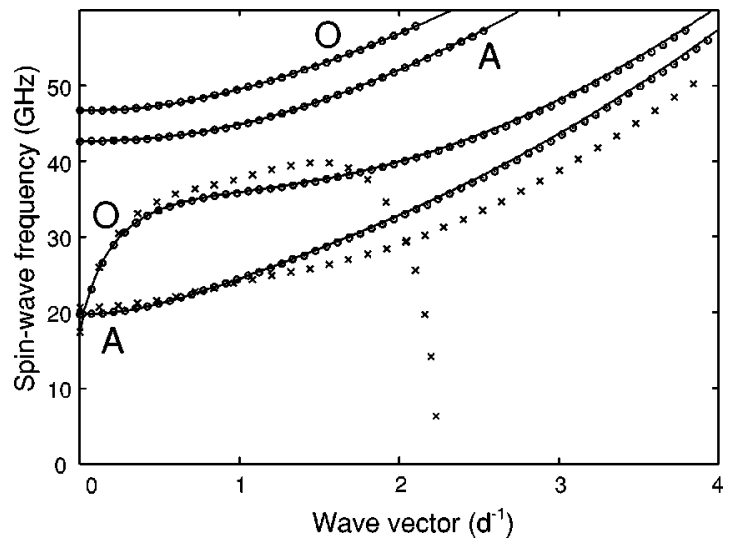

FIG. 5. Spin-wave frequencies in a Fe double layer as a function of $q d$. Solid lines, full calculation; crosses $(\times)$, conventional UTFA; open circles $(\bigcirc)$, extended UTFA with $\delta=2 \AA$. The two lower branches correspond to the surface $\mathrm{DE}$ and the higher branches correspond to the first standing modes. Used values: $d_{F e}$ $=200 \AA, J_{1}=1 \mathrm{~mJ} / \mathrm{m}^{2}, B=0.1 \mathrm{~T}$, all other parameters as in Fig. 1 .

\section{SAMPLE PREPARATION AND EXPERIMENTAL SETUP}

Epitaxial $\quad \mathrm{Fe}(80 \AA) / \mathrm{Si}$-wedge/Fe $(100 \AA) \quad$ trilayer samples are prepared by thermal electron-gun evaporation on top of a $\operatorname{GaAs}(001) / \mathrm{Fe}(10 \AA) / \mathrm{Ag}(1500 \AA)$ buffer system described in Ref. 25. Background pressure was better than $10^{-10}$ mbars. The thicknesses and the deposition rates of about $0.1 \AA / s$ for both $\mathrm{Fe}$ and $\mathrm{Si}$ are controlled by a calibrated quartz crystal monitor, and the layers are characterized by Auger electron spectroscopy, low-energy electron diffraction (LEED), and reflection high-energy electron diffraction (RHEED). The first $5 \mathrm{ML}$ of the bottom Fe layers are grown at room temperature (RT) in order to prevent segregation of $\mathrm{Ag}$ and the remainder at $200{ }^{\circ} \mathrm{C}$, which gives a good layer-by-layer growth according to the observed RHEED oscillations. The Si spacer and the top Fe layer are deposited at RT because higher temperatures lead to alloying of the spacer and a drastic reduction of the coupling strength. The well-defined LEED pattern observed throughout the whole structure indicates a good epitaxial growth. The samples are covered with a 500- $\mathrm{Z} \mathrm{ZnS}$ antireflection layer, which also prevents oxidation of the top Fe layer.

BLS experiments are performed using a Sandercock-type $(2 \times 3)$ pass tandem Fabry-Pérot interferometer ${ }^{26}$ in the backscattering geometry. The inelastically scattered light corresponding to both the Stokes (magnon creation) and antiStokes (magnon annihilation) processes is recorded using an avalanche diode detector and a multichannel analyzer PC card in the frequency range of $\pm 50 \mathrm{GHz}$. The wavelength $\lambda=532 \mathrm{~nm}$ of the laser light together with the incident angle of $45^{\circ}$ result in an in-plane magnon wave vector $q=1.67$ $\times 10^{7} \mathrm{~m}^{-1}$ of the measured magnons. The diameter of the laser spot on the sample of about $40 \mu \mathrm{m}$ is small enough compared to the slope of the wedge $(0.7 \AA / \mathrm{mm})$ to allow a precise measurement of the coupling strength. A variable external field with a maximum strength of $0.7 \mathrm{~T}$ is applied in the film plane and perpendicular to $\vec{q}$.
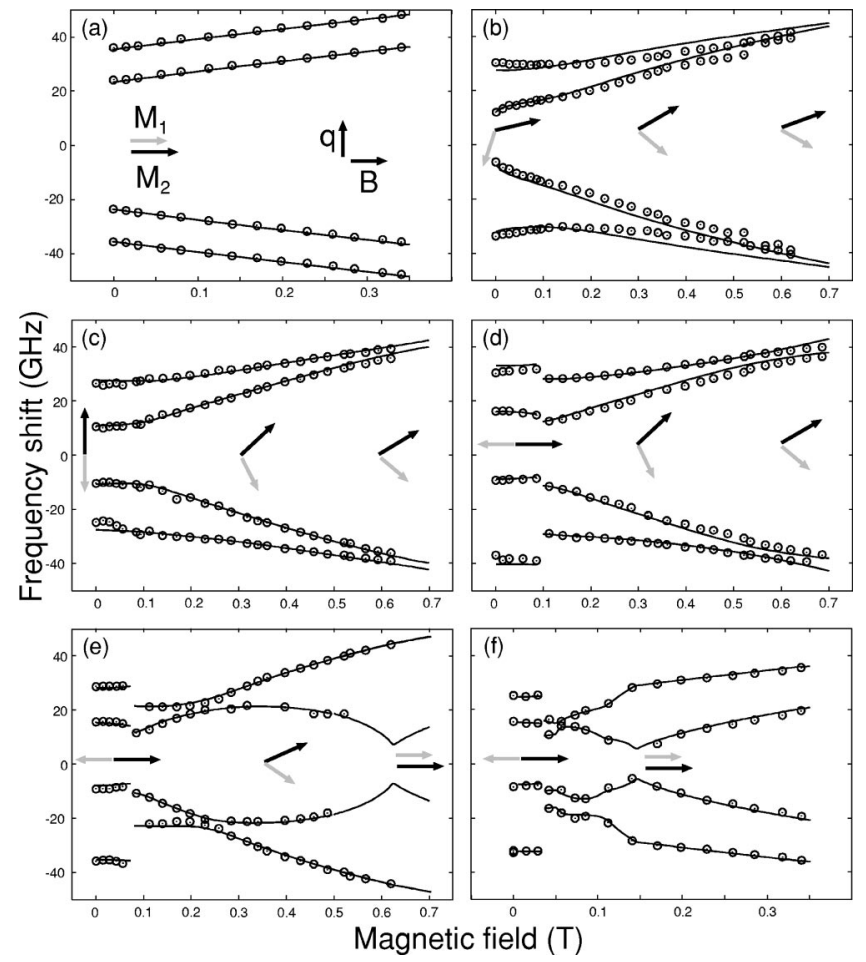

FIG. 6. Field dependence of measured BLS data (open circles) and least square fit (solid line) at different spacer thickness of a Fe $(80 \AA) /$ Si-wedge/Fe $(100 \AA)$ sample: (a) $4 \AA$, (b) $6 \AA$, (c) $7 \AA$, (d) $8 \AA$, (e) $10 \AA$, and (f) $12 \AA$. The coupling constants are given in the text. Arrows indicate the direction of the average magnetizations.

\section{RESULTS AND DISCUSSION}

In Fig. 6 we show typical field variation curves measured at different spacer thicknesses of a $\mathrm{Fe}(80 \AA) / \mathrm{Si}$ wedge/Fe $(100 \AA)$ sample with the field applied along an easy axis. The open circles are the experimental data and the solid lines correspond to a Levenberg-Marquardt fit using the EUTFA. We have used a sublayer thickness of $\delta=4 \AA$ for the calculation of both the twisted ground state and the magnon frequency and found a good enough precision compared to the accuracy of the frequency measurement of about 0.5 $\mathrm{GHz}$.

The coupling at small spacer thicknesses up to $5 \AA$ is FM and most likely due to direct exchange via pinholes. The value of the cubic anisotropy constant $K_{1}=45000 \mathrm{~J} / \mathrm{m}^{3}$ used to fit the data has been determined from the hard axis saturation field in the region of FM coupling. From the data in Fig. 6(a) we have derived a coupling strength of $J_{1}$ $=8.8 \mathrm{~mJ} / \mathrm{m}^{2}$ and a magnetization value $M_{S}=1.67$ $\times 10^{6} \mathrm{~A} / \mathrm{m}$. Here, as for all other fits, the perpendicular surface anisotropy of $\mathrm{Fe}(001)$ typically found to be of the order of $K_{s}=0.5 \mathrm{~mJ} / \mathrm{m}^{2}$ has been neglected. As the strength of surface anisotropy depends on the chemical environment and the morphology $y^{27,28}$ of the interface, it is likely to be different for all four interfaces and is therefore very difficult to measure. The perpendicular surface anisotropy has the approximate effect of reducing the magnetization derived from 


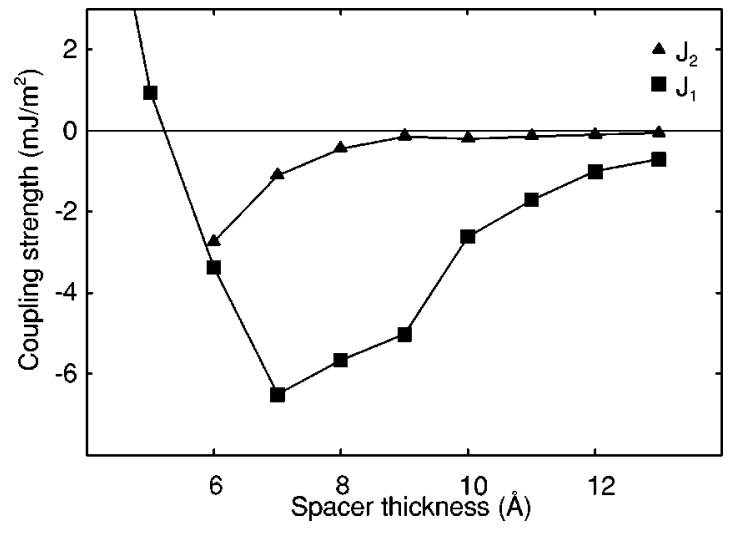

FIG. 7. Coupling constants as a function of spacer thickness.

a dynamic measurement by $2 K_{s} /\left(d \mu_{0} M_{s}\right)$, which here is of the order of $10 \%$.

The FM pinhole coupling decays quickly with increasing spacer thickness, and at an interlayer thickness of $6 \AA$ the coupling becomes predominantly biquadratic $\left(2\left|J_{2}\right|>\left|J_{1}\right|\right)$. This can be recognized in Fig. 6(b) by a distinct asymmetry between the Stokes and anti-Stokes sides, but without an AP state and the corresponding spin flop at low field. The coupling constants were found to be $J_{1}=-3.4 \mathrm{~mJ} / \mathrm{m}^{2}$ and $J_{2}$ $=-2.7 \mathrm{~mJ} / \mathrm{m}^{2}$, where $J_{2}$ is among the strongest biquadratic couplings measured so far.

The interlayer thickness region with dominant biquadratic coupling is very narrow. At a spacer thickness of $7 \AA$ in Fig. 6(c) $J_{1}$ has increased to $-6.5 \mathrm{~mJ} / \mathrm{m}^{2}$ while $J_{2}$ $=-1.1 \mathrm{~mJ} / \mathrm{m}^{2}$ is more than halved compared to Fig. 6(b). Interestingly, at low field the magnetizations are not in the ferrimagnetic ground state with the magnetizations collinear to the external field, but stay in an AP configuration perpendicular to the applied field, which has a higher free energy. Therefore, no spin flop can be identified in Fig. 6(c). For a field value of $50 \mathrm{mT}$, the ferrimagnetic state is calculated to be as much as $7 \times 10^{-5} \mathrm{~J} / \mathrm{m}^{2}$ lower in energy than the configuration found here. On the other hand, the pinning energy derived from the coercive field of the magnetization reversal at low field at a spacer thickness of $8 \AA$ has a value of only about $2.5 \times 10^{-5} \mathrm{~J} / \mathrm{m}^{2}$. Thus, the magnetizations are expected to flop into the ferrimagnetic state. A possible explanation for this unusual behavior is a nonisotropic form of the extrinsic biquadratic coupling as proposed by Slonczewski in Ref. 29.

The next graph in Fig. 6(d) with $8 \AA$ spacer thickness corresponds to the maximum of the optic-mode frequency in the AP state, which is related to the maximum of the effective coupling strength $J_{\text {eff }}$. The coupling constants are found to be $J_{1}=-5.66 \mathrm{~mJ} / \mathrm{m}^{2}$ and $J_{2}=-0.44 \mathrm{~mJ} / \mathrm{m}^{2}$. From here on both the bilinear and biquadratic coupling decay quickly until the sample decouples at about $15 \AA$ spacer thickness. The coupling constant in Figs. 6(e) and 6(f) with $10 \AA$ and $12 \AA$ spacer thickness are found to be $J_{1}=-2.61 \mathrm{~mJ} / \mathrm{m}^{2}$, $J_{2}=-0.20 \mathrm{~mJ} / \mathrm{m}^{2}, \quad$ and $\quad J_{1}=-1.00 \mathrm{~mJ} / \mathrm{m}^{2}, \quad J_{2}=$ $-0.10 \mathrm{~mJ} / \mathrm{m}^{2}$, respectively.

We have plotted $J_{1}$ and $J_{2}$ versus spacer thickness in Fig. 7. The strong biquadratic coupling $J_{2}$ at low thickness can be explained by the extrinsic fluctuation mechanism due to a competition of FM pinhole coupling and AFM interlayer coupling. The quick decay of $J_{2}$ with increasing spacer thickness is in agreement with this mechanism as the pinholes are expected to disappear quickly with increasing spacer thickness. The smooth decay of the intrinsic bilinear coupling $J_{1}$ is also in agreement with a very weak biquadratic coupling to the right of the AFM maximum, where $J_{2}$ is approximately $10 \%$ of $J_{1}$. A proportionality between $J_{1}$ and $J_{2}$ in the $\mathrm{Fe} / \mathrm{Si} / \mathrm{Fe}$ system has also been reported by Strijkers et al. ${ }^{30}$

The excellent agreement of the experimental data with the model calculation in Figs. 6(d)-(f), where $J_{2}$ is small, demonstrates that the magnetic configuration of the sample is close to the calculated ground state. In particular, the sample is in a single domain state and the twisted configuration conforms with the theory. The twisting angle in Fig. 6(c) at a field of $0.6 \mathrm{~T}$ comes out to be as big as $34^{\circ}$, which is comparable to the effects found in exchange springs. On the other hand, the poorer quality of the fit in Fig. 6(b) and the lack of the spin flop in Fig. 6(c), where $J_{2}$ is big, could be explained by the extrinsic origin of the biquadratic coupling. The fluctuation mechanism proposed by Slonczewski ${ }^{29}$ is supposed to have only to first order the biquadratic form and is probably even nonisotropic.

In earlier publications we have been using the conventional UTFA to extract the coupling constants from the field dependence of the BLS frequencies. The FM layers of the $\mathrm{Fe} / \mathrm{Si} / \mathrm{Fe}$ and $\mathrm{Fe} / \mathrm{Al} / \mathrm{Fe}$ samples investigated in Refs. 9 and 31 , respectively, were thinner, and the coupling of aluminum is weaker than that of silicon. Although the overall quality of the fits was good, the coupling constants especially for the strongest coupling derived in these publications have some systematic error. As discussed in Secs. II and IV, the UTFA with rigid magnetization approximation will underestimate $J_{\text {eff }}$ in the AP state and overestimate the biquadratic coupling strength when analyzing a full hysteresis loop. For the present sample, on the other hand, a satisfactory fit using the conventional UTFA is not possible except for Fig. 6(f) with rather weak coupling. The reason is the pronounced magnetization twist due to the thicker FM layers.

\section{CONCLUSIONS}

We have presented the EUTFA method based on continuum theory to calculate the spin-wave frequencies in coupled multilayers as measured by BLS and ferromagnetic resonance. Apart from a limitation to in-plane static magnetization and translational invariance in the direction parallel to the plane, arbitrary configurations including the canted and the twisted ground states can be taken into account. By comparing our model calculation with the results of the standard "full" approach and experimental data we have demonstrated that the method is accurate and well suited for the analysis of experimental data. From a fit to the field dependence of the BLS frequencies measured on a strongly AFM coupled $\mathrm{Fe} / \mathrm{Si} / \mathrm{Fe}$ sample, we have found strong evidence for the twisted ground state and derived the coupling constants as a function of the spacer thickness. The biquadratic contri- 


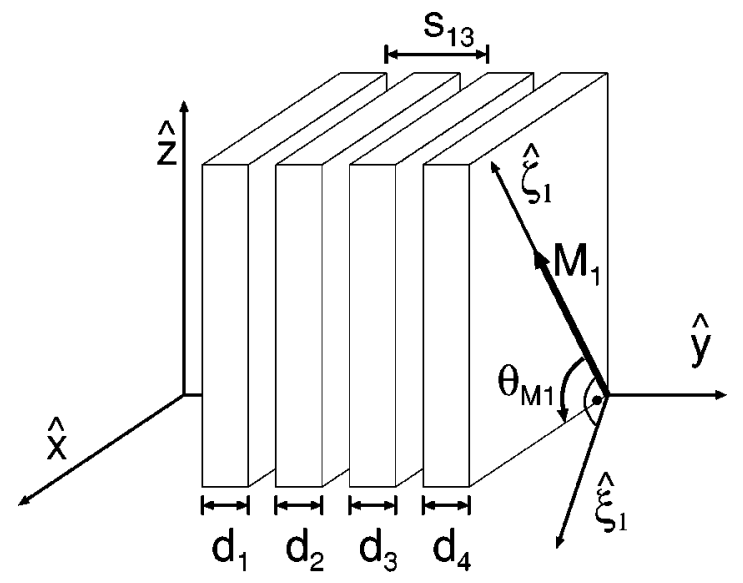

FIG. 8. Definition of the coordinate system. The $\hat{\zeta}_{i}$ and $\hat{\xi}_{i}$ axes are attached parallel and perpendicular to the static magnetizations $M_{i}$, respectively, and differ from layer to layer. $s_{i j}$ is the spacing between layers $i$ and $j$. All angles $\theta$ are in the plane and measured with respect to $\hat{x}$.

bution $J_{2}$ to the coupling is found to be dominant at a spacer thickness of $6 \AA$, where AFM coupling sets in. Then $J_{2}$ quickly decays with increasing spacer thickness to become about of $10 \%$ of $J_{1}$ to the right of the AFM maximum found at $7 \AA$. This evolution of the coupling constants with spacer thickness clearly supports the extrinsic origin of $J_{2}$ as proposed by Slonczewski.

Using our approach, an easy calculation of the spin-wave frequencies is possible even for much thicker samples up to about $100 \mathrm{~nm}$, for which a quantitative analysis previously only was possible in the rather limited cases of $P$ and AP configuration. Our approach could also be applied to exchange springs, where the scheme of Grimsditch et al. ${ }^{15}$ requires considerable computational effort.

\section{ACKNOWLEDGMENTS}

The authors would like to thank J. F. Cochran, R. L. Stamps, S. M. Rezende, M. Grimsditch, and B. Hillebrands for helpful discussions. This work was supported by the HGF-Strategiefondsprojekt "Magnetoelectronics."

\section{APPENDIX READY-TO-USE EQUATIONS FOR EUTFA}

We use a separate coordinate system $(\xi, y, \zeta)$ for each layer as in Ref. 7 and shown in Fig. 8. The local $z$ axis $\hat{\zeta}$ is attached to the static magnetization and the local $x$ axis $\hat{\xi}$ is in the plane and perpendicular to the static magnetization. The $y$ axis perpendicular to the film is common to all layers. The magnetic moments per unit area $\vec{m}_{i}$ in the local coordinate system are expressed by a coordinate transformation as a function of the magnetization $\vec{M}_{i}$ of layer $i$ in the absolute system $(x, y, z)$. Then $m_{i, \zeta}$ is the static part and $m_{i, \xi}$ and $m_{i, y}$ represent the small dynamic part of the magnetic moments. We consider, for each layer $i$, the Bloch equation with the effective field $\vec{b}_{i}^{e f f}$ acting on $\vec{m}_{i}$

$$
\gamma_{i}^{-1} d \vec{m}_{i} / d t=\vec{m}_{i} \times \vec{b}_{i}^{e f f} ; \quad b_{i, k}^{e f f}=-\left(\partial F / \partial m_{i, k}\right),
$$

where $k$ is the axis index, and $F$ is the free energy per unit area. The contributions to $b_{i, k}^{e f f}$ due to the Zeeman, anisotropy, and interlayer coupling energies are computed by expanding the free energy in terms of $m_{i, k}$, keeping only terms to quadratic order in $m_{i, \xi}$ and $m_{i, y}$. Only the contribution due to the dynamic dipolar coupling is not based on Eq. (A1) and more involved. ${ }^{7}$

Linearizing the Bloch equations and with the usual ansatz $\vec{m}_{i}(t)=\exp (i \omega t)\left(\hat{\xi} m_{i, \xi}+i \hat{y} m_{i, y}\right)+\hat{\zeta} m_{i, \zeta}$, one finally obtains a set of $2 N$ equations

$$
\sum_{i, k}\left(A_{j, l}^{i, k} m_{i, k}\right)=0 \quad(i, j=1, \ldots, N ; \quad k, l=y, \xi),
$$

where $N$ is the number of magnetic layers. The system of equations (A2) has solutions only for certain $\omega$, which are the spin-wave frequencies.

In the following, we will give the free energy terms and their corresponding terms of the matrix components $A_{j, l}^{i, k}$. Note that we have divided the $\xi$ rows by $i$ and the $y$ rows by -1 for convenience and in order to get real matrix elements.

\section{Zeeman energy}

$$
E_{Z}=\sum_{i} d_{i} \vec{M}_{i} \vec{B}_{e x t}
$$

leads to the matrix terms,

$$
A_{Z_{i, \xi}^{i, y}}=A_{Z_{i, y}^{i, \xi}}=B_{e x t} \cos \left(\theta_{M_{i}}-\theta_{B_{e x t}}\right) .
$$

\section{Cubic magnetocrystalline anisotropy}

$$
E_{c a}=\sum_{i} \frac{d_{i} K_{1 i}}{M_{i}^{4}}\left(M_{i, x}^{2} M_{i, y}^{2}+M_{i, x}^{2} M_{i, z}^{2}+M_{i, y}^{2} M_{i, z}^{2}\right) .
$$

Here, for simplicity, one easy axis is assumed to lie along the $\hat{x}$ direction. The following matrix terms take into account an arbitrary in-plane easy axis along $\hat{\theta}_{\text {ea }}$ :

$$
\begin{gathered}
A_{c a_{i, \xi}^{i, y}}=\frac{K_{1 i}}{2 M_{i}}\left[3+\cos \left\{4\left(\theta_{M_{i}}-\theta_{\mathrm{ea}}\right)\right\}\right], \\
A_{c a_{i, y}^{i, \xi}}=\frac{2 K_{1 i}}{M_{i}} \cos \left[4\left(\theta_{M_{i}}-\theta_{\mathrm{ea}}\right)\right] .
\end{gathered}
$$

\section{Uniaxial in-plane anisotropy}

$$
E_{u a}=-\sum_{i} \frac{d_{i} K_{u i}}{M_{i}^{2}}\left(\vec{M}_{i} \hat{\theta}_{\mathrm{ea}}\right)^{2}
$$

leads to the matrix terms 


$$
\begin{gathered}
A_{u a_{i, \xi}^{i, y}}=\frac{2 K_{u i}}{M_{i}} \cos ^{2}\left(\theta_{M_{i}}-\theta_{\mathrm{ea}}\right), \\
A_{u a_{i, y}^{i, \xi}}=\frac{2 K_{u i}}{M_{i}} \cos \left[2\left(\theta_{M_{i}}-\theta_{\mathrm{ea}}\right)\right] .
\end{gathered}
$$

\section{Surface anisotropy}

$$
E_{s a}=-\sum_{i} \frac{K_{s i}}{M_{i}^{2}} M_{i, y}^{2}
$$

leads to the matrix terms

$$
A_{s a_{i, \xi}^{i, y}}=-\frac{2 K_{s i}}{M_{i} d_{i}} .
$$

\section{Interlayer exchange coupling}

Note that the signs of $J_{1}$ and $J_{2}$ used by other authors might differ (e.g., Cochran et al. in Ref. 7 uses the opposite sign for $J_{2}$ ). We define the signs according to the generalized Heisenberg series $-\sum J_{i} \cos ^{i} \Delta \theta$ :

$$
\begin{aligned}
E_{C}= & -\sum_{i=1}^{N-1}\left[J_{1}^{i, i+1} \cos \left(\theta_{M_{i}}-\theta_{M_{i+1}}\right)\right. \\
& \left.+J_{2}^{i, i+1} \cos ^{2}\left(\theta_{M_{i}}-\theta_{M_{i+1}}\right)\right] .
\end{aligned}
$$

The corresponding matrix elements are

$$
\begin{aligned}
A_{C_{i, \xi}^{i, y}=} & \frac{1}{M_{i} d_{i}}\left[J_{1}^{i, i+1} \cos \left(\theta_{M_{i}}-\theta_{M_{i+1}}\right)+2 J_{2}^{i, i+1}\right. \\
& \times \cos ^{2}\left(\theta_{M_{i}}-\theta_{M_{i+1}}\right)+J_{1}^{i-1, i} \cos \left(\theta_{M_{i-1}}-\theta_{M_{i}}\right) \\
& \left.+2 J_{2}^{i-1, i} \cos ^{2}\left(\theta_{M_{i-1}}-\theta_{M_{i}}\right)\right], \\
A_{C_{i, y}^{i, \xi}=} & \frac{1}{M_{i} d_{i}}\left[J_{1}^{i, i+1} \cos \left(\theta_{M_{i}}-\theta_{M_{i+1}}\right)+2 J_{2}^{i, i+1}\right. \\
& \times \cos \left\{2\left(\theta_{M_{i}}-\theta_{M_{i+1}}\right)\right\}+J_{1}^{i-1, i} \cos \left(\theta_{M_{i-1}}-\theta_{M_{i}}\right) \\
+ & \left.2 J_{2}^{i-1, i} \cos \left\{2\left(\theta_{M_{i-1}}-\theta_{M_{i}}\right)\right\}\right], \\
A_{C_{i, \xi}^{i+1, y}=} & \frac{1}{M_{i+1} d_{i+1}}\left[-J_{1}^{i, i+1}-2 J_{2}^{i, i+1} \cos \left(\theta_{M_{i}}-\theta_{M_{i+1}}\right)\right], \\
A_{C_{i, \xi}^{i-1, y}=} & \frac{1}{M_{i-1} d_{i-1}}\left[-J_{1}^{i-1, i}-2 J_{2}^{i-1, i} \cos \left(\theta_{M_{i-1}}-\theta_{M_{i}}\right)\right], \\
A_{C_{i, y}^{i+1, \xi}=} \frac{1}{M_{i+1} d_{i+1}}\left[-J_{1}^{i, i+1} \cos \left(\theta_{M_{i}}-\theta_{M_{i+1}}\right)\right. & \left(\mathrm{A} 7 \mathrm{cos}\left\{2\left(\theta_{M_{i}}-\theta_{M_{i+1}}\right)\right\}\right],
\end{aligned}
$$

$$
\begin{aligned}
A_{C_{i, y}^{i-1, \xi}=} & \frac{1}{M_{i-1} d_{i-1}}\left[-J_{1}^{i-1, i} \cos \left(\theta_{M_{i-1}}-\theta_{M_{i}}\right)\right. \\
& \left.-2 J_{2}^{i-1, i} \cos \left\{2\left(\theta_{M_{i-1}}-\theta_{M_{i}}\right)\right\}\right] .
\end{aligned}
$$

\section{Dipolar terms}

Calculation of dipolar effective fields is more involved. We follow the approach by Cochran ${ }^{7}$ and evaluate the dipolar effective fields from the mode profiles in single-layer approximation, keeping terms second order in $q d_{i}$ as worked out by Rezende et al. ${ }^{14}$ This approximation is good as long as the thickness of all FM (sub)layers is small compared to the magnon wavelength; that means about $d_{i}<0.1 / q$ :

$$
A_{d i p}^{i, y}=\mu_{0} M_{i}\left(1-q d_{i} / 2\right)
$$

$$
\begin{gathered}
A_{d i p_{i, y}^{i, \xi}}=\frac{1}{2} \mu_{0} M_{i} q d_{i} \sin ^{2}\left(\theta_{M_{i}}-\theta_{q}\right), \\
A_{d i p_{i, \xi}^{j \neq i, y}=-\frac{1}{2}} \mu_{0} M_{i} q d_{i}\left(1-q d_{j} / 2\right) \exp \left(-q s_{i, j}\right), \\
A_{d i p_{i, y}^{j \neq i, \xi}=}=\frac{1}{2} \mu_{0} M_{i} q d_{i}\left(1-q d_{j} / 2\right) \exp \left(-q s_{i, j}\right) \\
\times \sin \left(\theta_{M_{i}}-\theta_{q}\right) \sin \left(\theta_{M_{j}}-\theta_{q}\right),
\end{gathered}
$$

$$
\begin{aligned}
A_{d i p_{i, \xi}^{j \neq i, \xi}=} & \frac{1}{2} \operatorname{sgn}(j-i) \mu_{0} M_{i} q d_{i}\left(1-q d_{j} / 2\right) \\
& \times \exp \left(-q s_{i, j}\right) \sin \left(\theta_{M_{j}}-\theta_{q}\right), \\
A_{d i p_{i, y}^{j \neq i, y}=} & -\frac{1}{2} \operatorname{sgn}(j-i) \mu_{0} M_{i} q d_{i}\left(1-q d_{j} / 2\right) \\
& \times \exp \left(-q s_{i, j}\right) \sin \left(\theta_{M_{i}}-\theta_{q}\right) .
\end{aligned}
$$

$s_{i, j}$ is the distance between layers $i$ and $j$ consisting of $|j-i|$ spacers and $|j-i|-1$ FM layers as sketched in Fig. 8.

\section{Intralayer exchange}

The effective fields due to the variation of the dynamic magnetization in the direction of the in-plane wave vector can be taken into account by ${ }^{13}$

$$
A_{e x_{i, \xi}^{i, y}}=A_{e x} x_{i, y}^{i, \xi}=D_{i} q^{2}
$$

where $D$ is the exchange stiffness constant defined in the usual way as $D=2 A / M$.

\section{Angular momentum}

Finally, the left-hand side of the Bloch equations is

$$
A_{\omega_{i, \xi}^{i, \xi}}^{i, \xi}=A_{\omega_{i, y}^{i, y}}=-\omega / \gamma_{i}
$$


*Electronic address: m.buchmeier@fz-juelich.de

${ }^{1}$ P. Grünberg, R. Schreiber, Y. Pang, M.B. Brodsky, and H. Sowers, Phys. Rev. Lett. 57, 2442 (1986).

${ }^{2}$ D. E. Bürgler, P. Grünberg, S. O. Demokritov, and M. T. Johnson, in Handbook of Magnetic Materials, edited by K. H. J. Buschow (Elsevier, Amsterdam, 2001), Vol. 13, pp. 1-85.

${ }^{3}$ van den Berg, W. Clemens, G. Gieres, G. Rupp, W. Schelter, and M. Vieth, IEEE Trans. Magn. 32, 4624 (1996).

${ }^{4}$ E.E. Fullerton, D.T. Margulies, M.E. Schabes, M. Carey, B. Gurney, A. Moser, M. Best, G. Zeltzer, K. Rubin, and H. Rosen, Appl. Phys. Lett. 77, 3806 (2000).

${ }^{5}$ S.S.P. Parkin and D. Mauri, Phys. Rev. B 44, 7131 (1991).

${ }^{6}$ J. Barnaś and P. Grünberg, J. Magn. Magn. Mater. 82, 186 (1989).

${ }^{7}$ J.F. Cochran, J. Rudd, W.B. Muir, B. Heinrich, and Z. Celinski, Phys. Rev. B 42, 508 (1990).

${ }^{8}$ F.C. Nörtemann, R.L. Stamps, and R.E. Camley, Phys. Rev. B 47, 11910 (1993).

${ }^{9}$ R.R. Gareev, D.E. Bürgler, M. Buchmeier, R. Schreiber, and P. Grünberg, J. Magn. Magn. Mater. 240, 237 (2002).

${ }^{10}$ B. Hillebrands, Phys. Rev. B 41, 530 (1990).

${ }^{11}$ R. Zivieri, L. Giovannini, and F. Nizzoli, Phys. Rev. B 62, 14950 (2000).

${ }^{12}$ B. K. Kuanr, M. Buchmeier, R. R. Gareev, D. E. Bürgler, R. Schreiber, and P. Grünberg, J. Appl. Phys. 93, 3427 (2003).

${ }^{13}$ R.L. Stamps, Phys. Rev. B 49, 339 (1994).

${ }^{14}$ S.M. Rezende, C. Chesman, M.A. Lucena, A. Azevedo, and F.M. de Aguiar, J. Appl. Phys. 84, 958 (1998).

${ }^{15}$ M. Grimsditch, R. Camley, E.E. Fullerton, S. Jiang, S.D. Bader, and C.H. Sowers, J. Appl. Phys. 85, 5901 (1999).

${ }^{16}$ E.F. Kneller and R. Hadwig, IEEE Trans. Magn. 27, 3588 (1991).

${ }^{17}$ R.W. Wang and D.L. Mills, Phys. Rev. B 50, 3931 (1994).

${ }^{18}$ L. Trallori, P. Politi, A. Rettori, M.G. Pini, and J. Villain, Phys. Rev. Lett. 72, 1925 (1994).

${ }^{19}$ W. Folkerts and S.T. Purcell, J. Magn. Magn. Mater. 111, 306 (1992).

${ }^{20}$ J.F. Cochran, J. Magn. Magn. Mater. 147, 101 (1995).

${ }^{21}$ S. Zoll, H.A.M. Van den Berg, K. Ounadjela, D. Stoeffler, and A. Dinia, J. Appl. Phys. 79, 2601 (1996).

${ }^{22}$ A. Hubert and R. Schäfer, Magnetic Domains (Springer, Berlin, 1998).

${ }^{23}$ William H. Press, Brian P. Flannery, Saul A. Teukolsky, and William T. Vetterling, Numerical Recipes in $C$ (Cambridge University Press, Cambridge, 2001), http://www.nr.com

${ }^{24}$ J.F. Cochran, Phys. Rev. B 64, 134406 (2001).

${ }^{25}$ A. Fuss, S. Demokritov, P. Grünberg, and W. Zinn, J. Magn. Magn. Mater. 103, L221 (1992).

${ }^{26}$ J.R. Sandercock, Top. Appl. Phys. 51, 173 (1982).

${ }^{27}$ B. Heinrich and J.F. Cochran, Adv. Phys. 42, 523 (1993).

${ }^{28}$ D.M. Schaller, D.E. Bürgler, C.M. Schmidt, F. Meisinger, and H.-J. Güntherodt, Phys. Rev. B 59, 14516 (1999).

${ }^{29}$ J.C. Slonczewski, Phys. Rev. Lett. 67, 3172 (1991).

${ }^{30}$ G.J. Strijkers, J.T. Kohlhepp, H.J.M. Swagten, and W.J.M. de Jonge, J. Appl. Phys. 87, 5452 (2000).

${ }^{31}$ B.K. Kuanr, M. Buchmeier, D.E. Bürgler, and P. Grünberg, J. Appl. Phys. 91, 7209 (2002). 